\title{
REAVALIAÇÃO DE FÓSSEIS DO GRUPO BAMBUÍ: IMPLICAÇÕES PALEOBIOLÓGICAS PARA O NEOPROTEROZOICO TARDIO DO BRASIL
}

\author{
Evelyn A. M. Sanchez ${ }^{1}$, Thomas R. Fairchild ${ }^{2}$ \\ 1- Centro de Estudos em Geociências, Instituto de Ciência e Tecnologia, Universidade Federal dos Vales do Jequitinhonha e Mucuri. \\ Rodovia MGT 367, km 583, no 5000. Alto da Jacuba, Diamantina, MG, CEP 39100-000. evelyn.sanchez@ict.ufvjm.edu.br \\ 2 - Departamento de Geologia Sedimentar e Ambiental, Instituto de Geociências, Universidade de São Paulo. \\ Rua do Lago, 562, Butantã, São Paulo, SP, CEP 05508-080. trfairch@hotmail.com
}

Recebido em 13 de janeiro de 2017; aceito em 11 de julho de 2017

\begin{abstract}
Resumo: Fósseis do Neoproterozoico tardio têm recebido grande atenção na última década por representarem profundas mudanças na biota. Tais mudanças incluem a passagem de uma biosfera dominada por formas procariontes unicelulares para formas eucariontes multicelulares. No Brasil, tem-se testemunhado muitos avanços no conhecimento sobre os fósseis desta idade, o que coloca o país na vanguarda das pesquisas paleontológicas do Neoproterozoico. Dentre as unidades brasileiras que figuram entre as que possuem este importante registro está o Grupo Bambuí, aflorante na porção central do Brasil. Fósseis têm sido identificados neste grupo desde o século XIX através de notas sobre o que hoje é conhecido como microbialitos, porém, foi na metade do século passado que o conhecimento sobre o registro fossilífero aumentou consideravelmente e passou a incluir possíveis icnofósseis, microfósseis e algas macroscópicas. No entanto, o significado destes fósseis tornou-se obsoleto, sobretudo mediante aos avanços da Paleontologia do Pré-Cambriano, ocorrida nas últimas duas décadas. Baseado na importância do registro fóssil do Grupo Bambuí e frente à eminente necessidade de sua contextualização no atual cenário de fósseis do Neoproterozoico, realizou-se uma reavaliação de fósseis descritos entre as décadas de 70 e 80 do século passado. Dos quatro táxons revistos, Kinneyia lucianoi Sommer 1970, Bambuilithos hectoris Sommer 1981 e Bambuilithos teixeranus Sommer 1982 passam a serem considerados pseudofósseis, enquanto que Bambuites erichsenii Sommer 1971 permanece classificado como morfofóssil, porém é posto em sinonímia com Leiosphaeridia jacutica (Timofeev, 1966), emend. Mikhailova \& Jankauskas, 1989. A reanálise desse material traz uma nova visão sobre a paleobiologia registrada no Grupo Bambuí e atualiza seu registro no panorama mundial de fósseis do Neoproterozoico tardio.
\end{abstract}

Palavras-chave: Grupo Bambuí, microfóssil, pseudofósseis, paleobiologia.

Abstract: RE-EVALUATION OF FOSSILS FROM BAMBUÍ GROUP: PALEOBIOLOGICAL IMPLICATIONS FOR THE LATE NEOPROTEROZOIC OF BRAZIL. Fossils of Late Neoproterozoic have received great attention in the last decade once they represent profound changes in biota. Such changes include the passage of a prokaryote-dominated biosphere to a multicellular, eukaryote-dominated biosphere. In Brazil, one has witnessed advances in knowledge concerning fossils of this age, placing the country at the forefront of paleontological research of the Neoproterozoic. Among the Brazilian units that comprise this important record is the Bambuí Group, outcropping in the central part of Brazil. Fossils have been identified in this unit since the eighteenth century via short notes about microbialites. However, it was in the middle of the 19th century that the knowledge of the fossil record has considerably increased, and then, included possible trace fossils, microfossils and macroscopic algae. Nonetheless, such record has become obsolete, mainly by the advances of Precambrian Paleontology, occurred in the last two decades. Based on the importance of the fossil record of the Bambui Group and through the imminent need for its context in the current Neoproterozoic fossils scenario, a re-evaluation of fossils described from the 70s and 80 s of last century was performed. Of the four groups reviewed, Kinneyia lucianoi Sommer 1970, Bambuilithos hectoris Sommer 1981 and Bambuilithos teixeranus Sommer 1982 are considered pseudofossils, and Bambuites erichsenii Sommer 1971 remained as a morphofossil, and was placed in synonymy with Leiosphaeridia jacutica (Timofeev, 1966), emend. Mikhailova \& Jankauskas, 1989. The re-analysis of this material provides new insight into the paleobiology recorded in the Bambuí Group and updates its record in the world panorama of the Late Neoproterozoic fossils.

Keywords: Bambuí Group, microfossil, pseudofossils, paleobiology

\section{INTRODUÇÃO}

O Grupo Bambuí é uma das principais coberturas sedimentares do Neoproterozoico do Brasil. Dados de subsuperfície demonstraram que este grupo foi depositado em sistemas de grabens em ambientes de forebulge (Reis \& Suss, 2016), e é caracterizado por reunir um registro misto de rochas carbonáticas e siliciclásticas depositadas em resposta ao estabelecimento de um regime tectônico de foreland frente à amalgamação da porção oeste de Gondwana, variações eustáticas e às mudanças climáticas estabelecidas durante e após um dos episódios glaciais do Neoproterozoico tardio (Alkmin \& Martins-Neto, 2012; Caxito et al., 2012; Reis \& Suss, 2016). Dados de diferentes naturezas apontam uma idade Neoproterozoica tardia para o Grupo Bambuí. Caxito e colaboradores (2012) identificaram uma capa carbonática Marinoana, depositada após um dos episódios glaciais neoproterozoicos, na Formação Sete Lagoas, base do grupo, atestando idade de 635 Ma. Datações com base em zircões detríticos, provenientes de vários pontos da bacia, sugerem que sua deposição ocorreu por volta de 600 a 650 Ma (Rodrigues, 2008) e teria adentrado no Cambriano, havendo dados que sugerem que todo o grupo foi depositado após 550 Ma (Kuchenbecker, 2014). Este mesmo cenário condiz com a ocorrência do macrofóssil Cloudina sp. na Formação Sete Lagoas (Warren et al., 2014), cuja ocorrência é mundialmente datada do fim do Ediacarano, em 542 Ma (Grant, 1990). 
Do ponto de vista paleontológico, o Grupo Bambuí apresenta grande potencial fossilífero (Bittencourt et al., 2016), uma vez que foi depositado em um intervalo de tempo em que a biosfera passou por diversas inovações evolutivas (figura 1) nas mais variadas escalas, desde a diversificação do plâncton marinho (Grey, 2005), a origem dos metazoários e a inovação de relações ecológicas e de ecossistemas (Xiao \& Laflamme, 2009), todos influenciados por mudanças ambientais diversas (Fedonkin, 2003; Sperling et al., 2013; Mills \& Canfield, 2014).

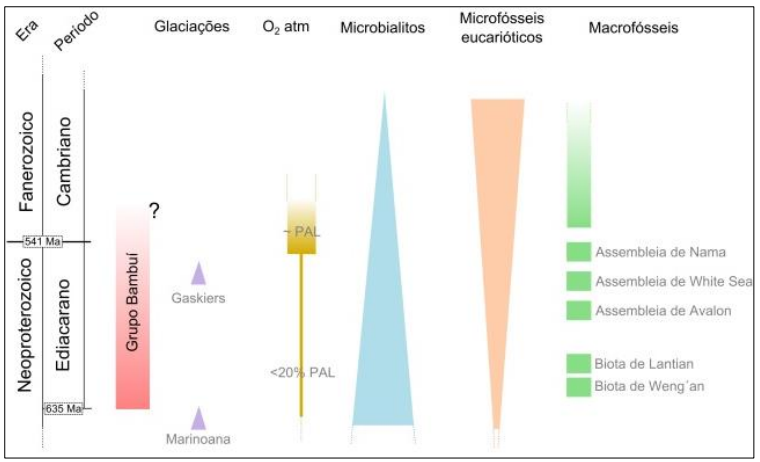

Figura 1. Contextualização do Grupo Bambuí no panorama mundial de fósseis do Ediacarano (Neoproterozoico Tardio) e em relação à ocorrência de glaciações, as quais são apontadas como fator importante de aumento de diversidade após $635 \mathrm{Ma}$. Dos elementos mostrados na imagem, apenas a glaciação Gaskiers ainda não foi identificada no registro do grupo. Dados compilados de Knoll (2003) e Schopf (1992). PAL = present atmospheric levels.

Atualmente, o registro paleontológico do Grupo Bambuí, sobretudo em sua porção basal, a Formação Sete Lagoas, inclui diversos microbialitos (Sanchez, 2014), microfósseis procarióticos silicificados (Fairchild et al., 1996), alguns acritarcos (Hidalgo, 2007), os metazoários Cloudina sp. e Corumbella sp. associados a icnofósseis (Warren et al., 2014) e algumas estruturas enigmáticas, descritas há décadas, e cuja biogenicidade permanece incerta (Sommer, 1970, 1971, 1981, 1982). São estas estruturas que compõem o cerne do presente estudo.

Ao longo do século passado, a Paleontologia e muitos de seus conceitos foram refinados, principalmente à medida que novas tecnologias, tais como Espectrometria Raman e Microtomografia de Raios- $X$, foram incorporadas aos métodos, o que levou à reavaliação de vários grupos fósseis, à reinterpretação de afinidades biológicas e de seus significados evolutivo e bioestratigráfico, inclusive aqueles oriundos do Proterozoico (i.e. Tafforeau et al., 2006; Chen et al., 2009; Morris \& Caron, 2012). Frente a tais avanços da paleobiologia do PréCambriano e à luz de técnicas mais recentemente aplicadas em Paleontologia, fez-se necessário reavaliar algumas ocorrências fósseis no Grupo Bambuí, devido à importância do grupo. Os fósseis aqui analisados estão tombados na coleção do DNPM no Rio de Janeiro desde que foram descritos, nas décadas de 1970 e 1980 . A reavaliação destas ocorrências sob a luz de conhecimentos atualizados será importante para i) estabelecer se, de fato, estas estruturas são fósseis para então ii) agregar valor bioestratigráfico, paleoambiental e evolutivo a eles e, por fim, iii) demonstrar sua utilidade para entender em qual contexto paleobiológico algumas seções fossilíferas do Grupo Bambuí foram depositadas.

\section{MATERIAL E MÉTODOS}

As amostras aqui analisadas foram coletadas, identificadas e publicadas entre 1970 e 1982, e estão tombadas na coleção científica de invertebrados fósseis e no laminário do Departamento Nacional de Produção Mineral na cidade do Rio de Janeiro (DNPM-RJ). Trata-se de amostras de mão (Tabela 1) e uma lâmina palinológica, cuja proveniência exata é desconhecida, sabendo-se apenas os municípios onde foram coletadas, sendo Pitangui e Pedro Leopoldo, ambos em Minas Gerais (figura 2). Estas amostras foram coletadas em calcarenitos e ardósias, litologias comuns nas formações que compõem o Grupo Bambuí. Por esta razão, não foi possível atribuir um posicionamento estratigráfico para as amostras.

Tabela 1. Fósseis encontrados em afloramentos do Grupo Bambuí, em Minas Gerais, depositados no acervo do DNPM/RJ.

\begin{tabular}{c|c|c|c|c} 
Fóssil & $\begin{array}{c}\text { Números de } \\
\text { tombo } \\
\text { DNPM-RJ }\end{array}$ & $\begin{array}{c}\text { Interpretação } \\
\text { original }\end{array}$ & $\begin{array}{c}\text { Local de } \\
\text { Coleta }\end{array}$ & Autor \\
\hline $\begin{array}{c}\text { Kinneyia } \\
\text { lucianoi }\end{array}$ & $\begin{array}{c}1683-\mathrm{Pb} \text { a } \\
1687-\mathrm{Pb}\end{array}$ & $\begin{array}{c}\text { Icnofóssil de } \\
\text { algas talófitas }\end{array}$ & $\begin{array}{c}\text { Município de } \\
\text { Pedro } \\
\text { Leopoldo }\end{array}$ & $\begin{array}{c}\text { Sommer } \\
(1970)\end{array}$ \\
\hline $\begin{array}{c}\text { Bambuites } \\
\text { erichsenii }\end{array}$ & $\begin{array}{c}\text { Lâmina não } \\
\text { tombada }\end{array}$ & $\begin{array}{c}\text { Vestígios de } \\
\text { algas }\end{array}$ & $\begin{array}{c}\text { Município de } \\
\text { Pedro } \\
\text { Leopoldo }\end{array}$ & $\begin{array}{c}\text { Sommer } \\
(1971)\end{array}$ \\
\hline $\begin{array}{c}\text { Bambuilithos } \\
\text { hectoris }\end{array}$ & $1688-\mathrm{Pb}$ & Oncólito & $\begin{array}{c}\text { Município de } \\
\text { Pintangui }\end{array}$ & $\begin{array}{c}\text { Sommer } \\
(1981)\end{array}$ \\
\hline $\begin{array}{c}\text { Bambuilithos } \\
\text { teixeranus }\end{array}$ & $1689-\mathrm{Pb}$ & Oncólito & $\begin{array}{c}\text { Município de } \\
\text { Pedro } \\
\text { Leopoldo }\end{array}$ & $\begin{array}{c}\text { Sommer } \\
(1982)\end{array}$ \\
\hline
\end{tabular}

A análise do material de mão contou com o uso de lentes de aumento e imagens de alta resolução. Já a análise da lâmina palinológica foi realizada com - auxílio de microscópio biológico marca Zeiss, modelo Axio Imager acoplado a um sistema de captura de imagem AxionVision 4.8, também da marca Zeiss. 


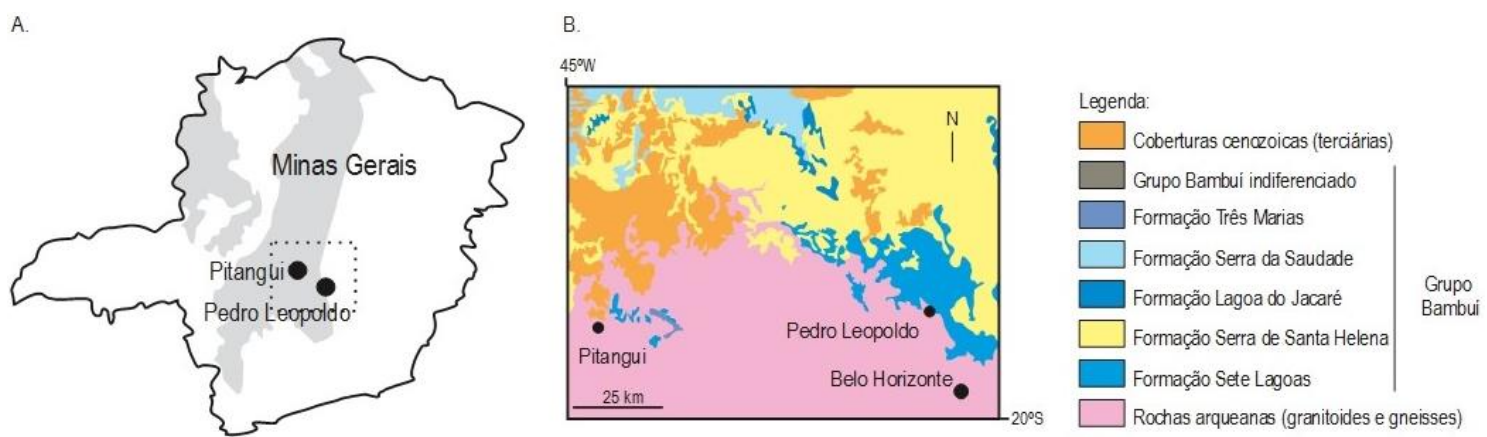

Figura 2. A) Ocorrência do Grupo Bambuí em Minas Gerais e localidades onde as estruturas analisadas no presente trabalho foram recuperadas. Polígono pontilhado corresponde ao mapa detalhado em B. B) Mapa geológico das localidades onde o material analisado foi encontrado (CPRM, 2014).

\section{REINTERPRETAÇÃO DOS FÓSSEIS DO GRUPO BAMBUÍ}

\section{Gênero Bambuilithos}

Entre 1981 e 1982, Sommer identificou duas estruturas nodulares em calcários do Grupo Bambuí, as quais classificou como algas talófitas Bambuilithos hectoris Sommer 1981 e Bambuilithos teixeranus Sommer 1982, respectivamente. Na ocasião da publicação estes fósseis foram atribuídos ao subgrupo Oncolithi Pia 1927, que, junto com o subgrupo Stromatolithi, compunha a família de cianobactérias Spongiostromata, uma subdivisão da classe Talófitas (Pia, 1927). No entanto esta nomenclatura não é mais utilizada, e o termo Spongiostromata ficou restrito à denominação de microtextura de microbialitos, composta por laminações que incluem uma série de características, entre elas micrita ou esparita, peloides, material detrítico, fenestras e textura esponjosa (Monty, 1981).

O holótipo de $B$. hectoris Sommer 1981 (figura $3 A-D)$ é composto de três partes que, quando unidas, têm aparência nodular. O holótipo encontrase tombado sob o código 1688-Pb/DNPM-RJ.

A estrutura esférica foi formada pela silicificação de calcário e apresenta coloração cinza escura e brilho ceroso (figuras 3A-B). Ocorrem duas texturas, uma na metade superior e outra na metade inferior. A porção inferior (figura 3B) tem aspecto maciço, sem estrutura sedimentar ou diagenética aparente, apenas moldes de alguns clastos arredondados. Já a parte superior (figura 3A) é marcada pelo padrão Kinneyia e atravessa três níveis de alguns centímetros de espessura, que originalmente eram camadas. As mesmas cristas atravessam toda a extensão da parte superior deste nódulo. Estas cristas são retas, raramente curvas, e chegam a alcançar $4 \mathrm{~cm}$ de extensão, cobrindo toda a parte superior do nódulo. Na lateral desta amostra há uma porção que não foi afetada pelo tratamento ácido que Sommer utilizou para realçar as estruturas do espécime (figura 3C). No entanto, está altamente intemperizada e apresenta moldes de grãos de diferentes tamanhos, com bordas arredondadas, densamente empacotados. O padrão Kinneyia não foi observado.

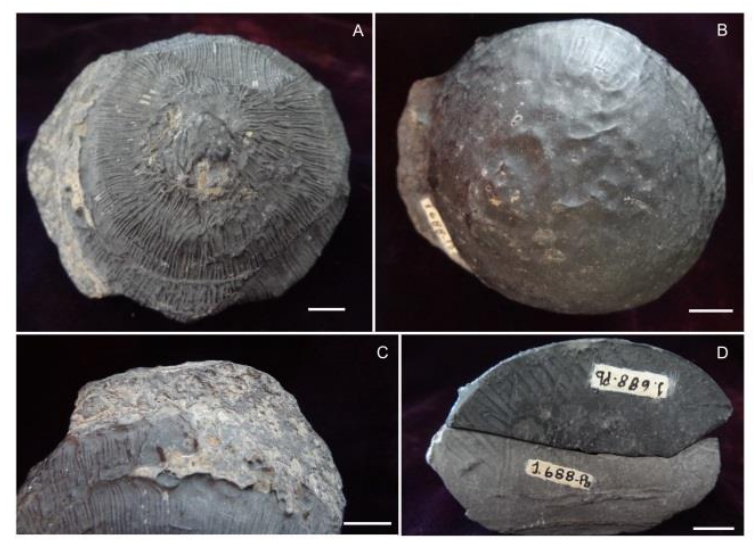

Figura 3. Bambuilithos hectoris Sommer 1981, amostra 1688-Pb. A: vista em planta do nódulo, apresentando o padrão de cristas. Notar que as mesmas cristas cortam diferentes camadas. B: Porção inferior com aspecto maciço. C: Resto do material que cobria a amostra. Presença de moldes de grãos e coloração típica de material intemperizado. D: Outros fragmentos da mesma amostra. Notar que as cristas estão localizadas somente na superfície da metade superior, não havendo relação com as camadas que formam o nódulo. Escalas: $1 \mathrm{~cm}$.

Em resumo, os caracteres que definem o fóssil Bambuilithos hectoris reúnem três conceitos paleontológicos que não ocorrem juntos no registro paleontológico: $B$. hectoris foi classificado como um i) oncoide ou nódulo do grupo das ii) talófitas e apresenta padrão de iii) estrutura microbiana Kinneyia.

Oncoides são grãos envelopados, de natureza carbonática, compostos de multilamelas (córtex) que envolvem um núcleo de natureza diversa. A origem do córtex está ligada à precipitação de carbonato pela ação de tapetes cianobacterianos que circundam o núcleo, dando ao oncoide aspecto 
concêntrico (Flügel \& Axel, 2010). Esta característica não está presente em $B$. hectoris.

Já registros de Kinneyia (figura 4) ocorrem em rochas siliciclásticas. Estas estruturas foram amplamente discutidas por Porada \& Bouougri (2007) e foram atribuídas a microorganismos colonizadores de superfícies ricas em grãos silicilásticos. São caracterizadas por cristas de topo plano, curvas, paralelas entre si, alternadas com escavações na superfície, lembrando marcas onduladas (ripples) de pequena escala. As cristas podem se bifurcar ou se unir e formar estruturas semelhantes a colmeias. Entretanto, neste mesmo trabalho, os autores apontam para a possibilidade de confundir a estrutura Kinneyia com outras marcas de tração, tal como estruturas de sobrecarga, marcas onduladas de adesão, entre outras.
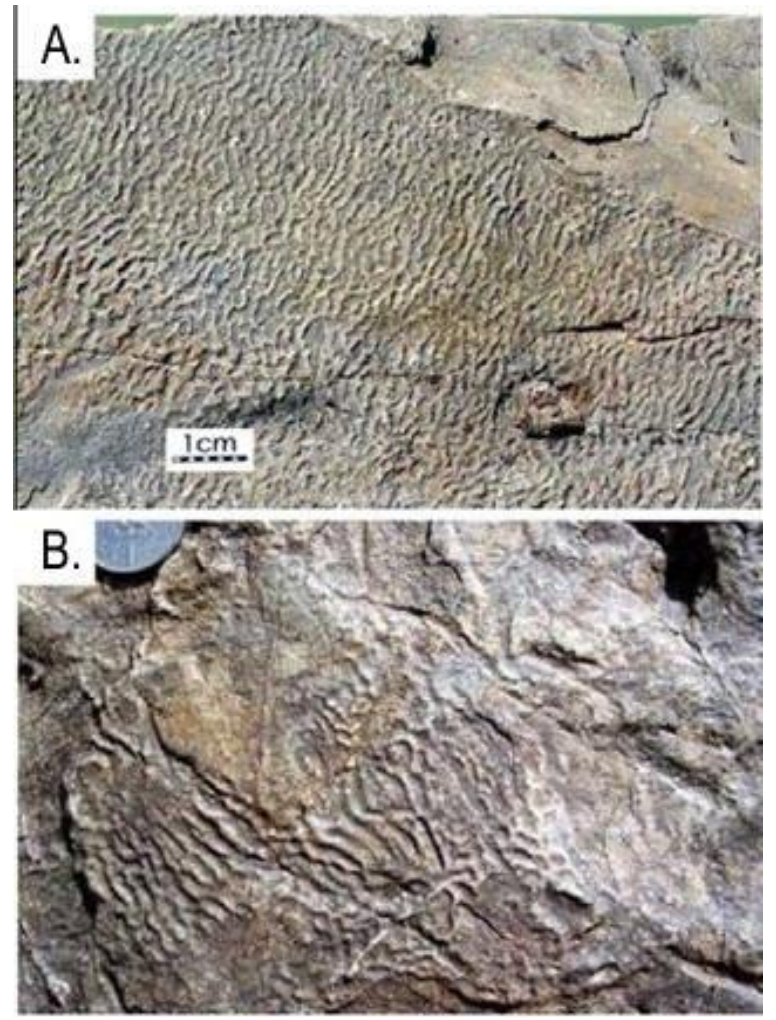

Figura 4. Estruturas Kinneyia preservadas em superfícies de acamamento (imagens de Porada \& Bouougri, 2007). A) amostra do siltito Paradoxissimus, localidade de Oeland, Suécia,

Cambriano Médio. B) amostra coletada na Formação Wanimzi, Grupo Tizi n-Taghatine, Marrocos, Neoproterozoico. Moeda $=24$

$\mathrm{mm}$ diâmetro. Notar cristas retilíneas e arredondadas

semelhantes à Bambuilithos hectoris, no entanto preservada em uma superfície de acamamento.

No caso de Bambuilithos hectoris, as cristas são retas e não apresentam o perfil ondulado como esperado para Kinneyia. Outra característica notória é que as cristas são oblíquas ao acamamento, atravessando, pelo menos, três camadas finas, ao passo que o padrão de Kinneyia ocorre apenas na superfície de acamamento.

No que tange à atribuição de Bambuilithos às talófitas, esta situação não condiz com a história geológica deste grupo. As talófitas, grupo artificial que reúne algas, líquens, briófitas e fungos, têm o registro mais antigo (figura 5 ) encontrado na Formação Doushantuo, na província de Guizhou, no sul da China (Zhang, 1989). Estes espécimes apresentam tamanho centimétrico e diferenciação de tecidos em sua parte interna. Devido ao seu tamanho diminuto poucos centímetros, só puderam ser identificados através de lâminas petrográficas em amostras de fosforitos (Zhang, 1989). A parte externa das talófitas fósseis, conhecida como córtex, é composta por uma camada de $100 \mu \mathrm{m}$ de células alongadas, carecendo do padrão Kinneyia, como ocorre em Bambuilithos. Já a parte interna, chamada de medula, é composta uma série de células de diferentes formas, organizadas em ilhas. A porção interna de Bambuilithos hectoris é lisa, com brilho ceroso, e não apresenta qualquer tipo de estrutura que remeta à diferenciação de tecidos.

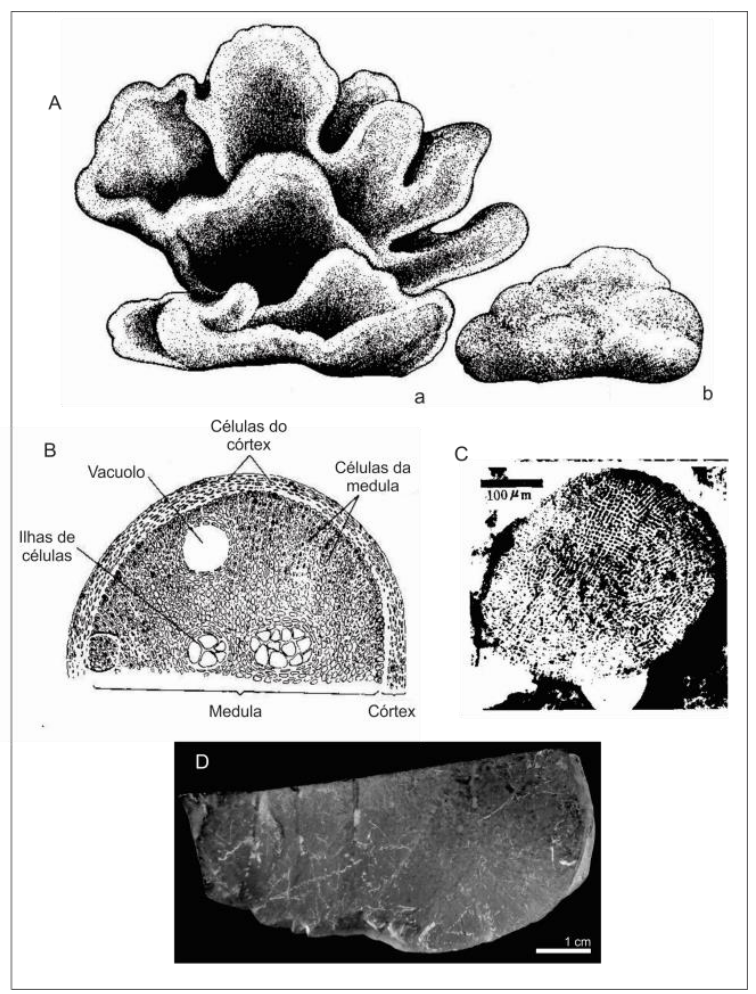

Figura 5. Talófitas fósseis. A) reconstituição do gênero Thallophyca Zhang 1989 a partir de amostras coletadas na Formação Doushantuo, China (ca. 630 Ma). a, T. ramosa e b, T. simplica. Desenho sem escala. B) desenho esquemático mostrando a anatomia do gênero Thallophyca. C) Wengania globosa Zhang 1989, outro gênero de talófita encontrado na Formação Doushantuo, China. D) parte interna da Bambuilithos hectoris mostrando superfície lisa, sem diferenciação de tecido. Imagens A-C a partir de Zhang (1989). 
Deve-se ressaltar que Bambuilithos hectoris também apresenta algumas características que levantam dúvida sobre sua biogenicidade. Entre as características que apontam para origem inorgânica estão o modo de ocorrência das cristas e a necessidade de tratamento químico. Para revelar as cristas foi necessário que as amostras passassem por tratamento com ácidos. Não se pode descartar que os ácidos, ao atacarem o carbonato da amostra, pudessem gerar estruturas, tais como as cristas retas, raramente curvadas, e simétricas, o que não está de acordo com a típica simetria do padrão Kinneyia. Adicionalmente, as cristas não ocorrem no plano de acamamento como esperado para o padrão Kinneyia, mas, em, pelo menos, três laminações.

Por tanto, a partir destes argumentos e, ao reunir os dados da literatura sobre oncoides, talófitas e Kinneyia, pode-se concluir que a única característica restante é que Bambuilithos hectoris remete a um nódulo sendo, portanto, um pseudofóssil resultante do tratamento ácido ao qual a amostra foi submetida.

A outra espécie, Bambuilithos teixeiranus Sommer 1982, apresenta características totalmente diferentes de $B$. hectoris. A justificativa do autor para atribuir o holótipo ao gênero Bambuilithos seria o fato de tratar-se de um oncoide.

A única amostra desta espécie está preservada em uma matriz composta por calcarenito branco, tendo granulometria média na base e na porção superior, e grãos mais grossos na porção mediana (figuras 6A-B). Na porção intermediária ocorrem estruturas semelhantes a nódulos, compostas de sílex cinza escuro, algumas preservadas tridimensionalmente, e outras com apenas uma face exposta, podendo ser envolvidas por um envelope (figura 6F). Uma das estruturas exposta tridimensionalmente permite observar um formato achatado no eixo longitudinal, com cerca de $1 \mathrm{~cm}$ de espessura. $\mathrm{Na}$ superfície, apresenta sulcos concêntricos (figura 6E).

Por sua forma arredondada, quase discoide (figura 6D-E), e pelas características da superfície (figura 6E) levaram à formulação de duas hipóteses para explicar $B$. teixeiranus: uma hipótese paleontológica, que permite assumir que esta estrutura seria um fóssil, confirmando as ideias de Sommer (1982), ou a hipótese abiogênica, em que $B$. teixeiranus consiste de nódulos de sílex, semelhante a aqueles descritos por Seilacher (2001), sendo, portanto, um pseudofóssil. Descarta-se aqui a hipótese de serem clastos de ooides ou oncoides devido à sua morfologia discoide.

No primeiro caso, a ocorrência de vários espécimes semelhantes, com tamanho variado, apontaria positivamente para uma interpretação biológica para $B$. teixeiranus. Já a forma vesicular, ligeiramente achatada, permite inferências sobre a afinidade biológica desta espécie. Formas vesiculares do Neoproterozoico tardio, de tamanho centimétrico e carente de qualquer estrutura interna, têm sido atribuídas aos vendobiontes. Trata-se de grupo fóssil restrito ao Neoproterozoico terminal, cuja afinidade biológica é incerta, embora algumas propostas já tenham sido feitas, entre elas trata-se de um grupo sem descendência conhecida (Seilacher, 1992), ou são um grupo correlato metazoários (Bruss \& Seilacher, 1994), ou ainda são protozoários unicelulares de grandes dimensões (Seilacher, 2007).

Dentre os vendobiontes vesiculares com simetria radial, tal qual B. teixeiranus, desponta o gênero Nemiana, cuja única espécie é $N$. simplex (Leonov, 2007). Esta espécie já foi reconhecida nas formações ediacaranas Yampol e Mogilev, na Polônia, na Formação Yorga, região de White Sea, na Rússia, na sequência sedimentar das montanhas Wemecke, no Canadá, e na Formação Dabis, na Namíbia. Consistem de impressões em alto relevo, com forma de vesícula, típicas de ambientes areníticos, sendo os melhores exemplares preservados na interface argila-arenito (Leonov, 2007). Acredita-se que Nemiana tenha sido um organismo bentônico sedentário, que vivia parcialmente soterrado em fundos lamosos, em assembleias monoespecíficas de vários indivíduos (figura 6l), de tamanhos variados. Seu relevo alto deve-se à preservação por rápido soterramento e preenchimento dos espécimes com sedimentos, sendo que na diagênese poderia ser compactada, gerando deformação no substrato subjacente (Leonov, 2007).

Ao se comparar as diagnoses de Nemiana e Bambuilithos, uma possível sinonímia no nível de gênero poderia ser proposta, já que ambos consistem de vesículas centimétricas, preservadas em ambiente clástico. No entanto, em nível de espécie não é possível igualar os dois táxons, pelo fato de Bambuilithos teixeiranus apresentar sulcos concêntricos em sua superfície, ao passo que Nemiana simplex não apresenta qualquer ornamentação, apenas marcas de achatamento durante a preservação (Leonov, 2007). 

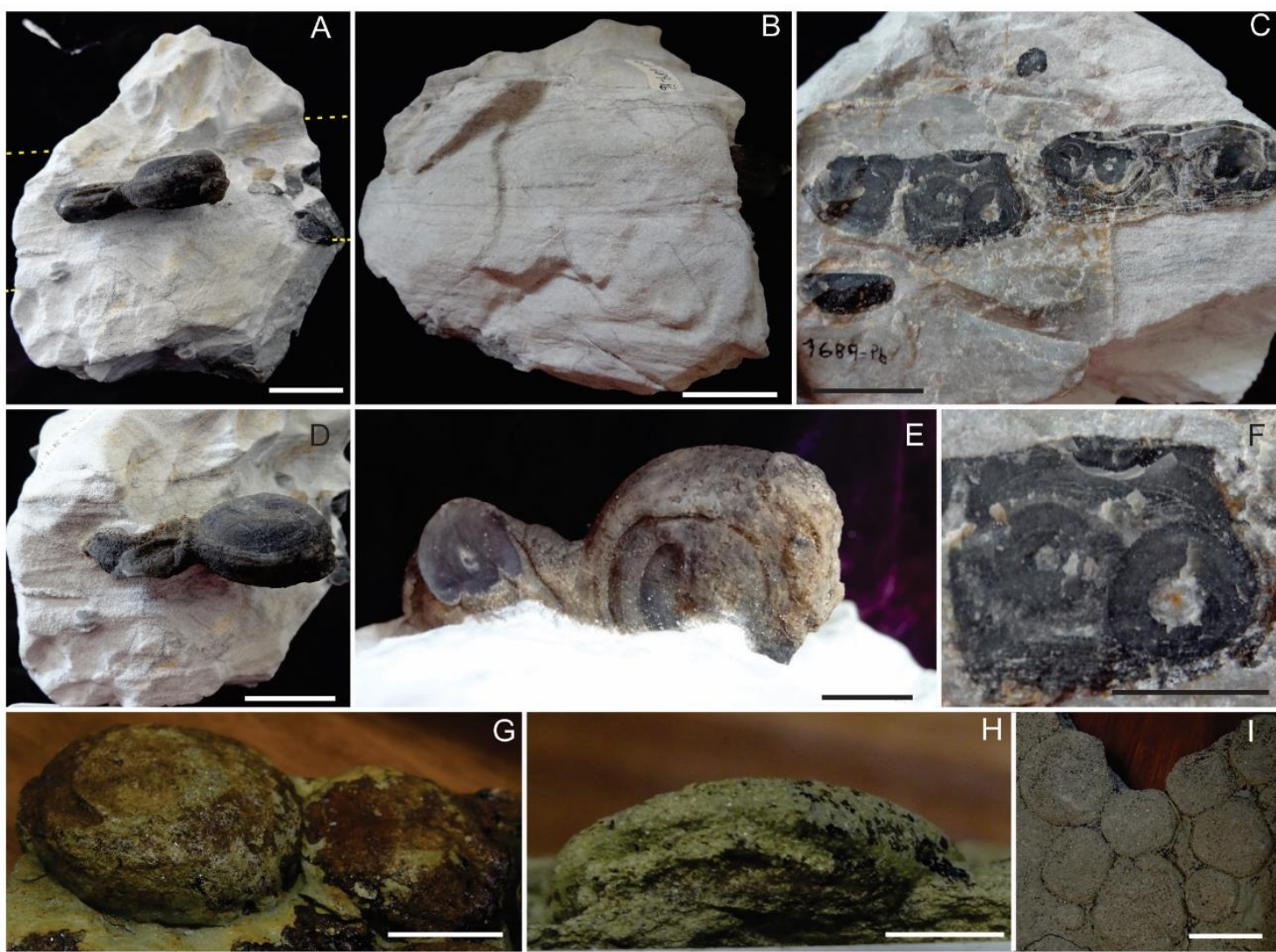

Figura 6. A-F) Bambuilithos teixeiranus (holótipo 1689-Pb), Grupo Bambuí. G-I) Nemiana simplex, assembleia de White Sea, Rússia (coleção didática de Oxford University). Em A as linhas amarelas marcam os limites entre os níveis de calcarenito médio nas extremidades da amostra e de calcarenito grosso na faixa central. B) Face sem indivíduos de B. teixeiranus. C) Clastos centimétricos alinhados, com indivíduos de B. teixeiranus. D) Detalhe dos dois espécimes preservados tridimensionalmente. E) Detalhe da superfície de um dos espécimes. Sulcos seguem de maneira homogênea o contorno circular da amostra, aparentando serem primários. F) Detalhe dos espécimes preservados em duas dimensões, que não apresentam laminação interna. Setas indicam estrutura que envolve os espécimes, semelhante a um envelope. G, I) Detalhe da superfície de Nemiana simplex e a relação entre os indivíduos. H) Corte longitudinal de $N$. simplex, que não foi possivel verificar em $B$. teixeiranus.

Em vista do exposto, a interpretação restante é que $B$. teixeiranus consiste de um conjunto de nódulos, invocando, portanto, uma origem abiogênica e diagenética. Segundo o modelo proposto por Seilacher (2001), a forma ovóide, seria uma resposta à compactação do sedimento, ainda na fase eodiagenética, que resulta na diminuição da permeabilidade na direção vertical, consequentemente alterando a cimentação. Neste trabalho, Seilacher aponta para algumas estruturas que foram erroneamente classificadas como fósseis (e.g. Palaeotrochis Häntzschel, 1975), mas que, na verdade, eram nódulos. É provável que, se a origem puramente química se aplicar à gênese de Bambuilithos teixeiranus, este evento ocorreu em outra camada, que, após a precipitação e litificação dos nódulos de sílex, foi retrabalhada e os nódulos foram redepositados como clastos em meio ao calcarenito. A evidência de retrabalhamento está no fato de que os nódulos apresentam composição química (sílex), textural e de cor (cinza escuro) diferente da matriz que os contém.

\section{Kinneyia lucianoi}

Em 1970, Sommer identificou estruturas provenientes da pedreira da fábrica de cimento Cauê, próximo à Pedro Leopoldo, e atribuiu-lhes origem biológica, provavelmente algas da espécie Kinneyia lucianoi. No entanto, as amostras foram, segundo o autor, submetidas à tratamento com alguns ácidos, porém não especificados, para ressaltar as estruturas de interesse, chamadas por ele de bióglifos.

Cinco exemplares de Kinneyia lucianoi fazem parte do acervo paleontológico do DNPM. Trata-se de fragmentos carbonáticos, poligonais, planos, não nodulares de tamanho centimétrico. A característica mais proeminente, como esperado para esta espécie, é a ocorrência de cristas (figuras 7A-D), tais qual aquelas de $B$. hectoris, inclusive obtidas da mesma maneira, através do tratamento com ácidos. Chama a atenção o fato de que as cristas de $K$. lucianoi passam através de estruturas secundárias, como é o caso na amostra $1683-\mathrm{Pb}$, em que as cristas passam através de uma lente de calcita recristalizada (figura 7B). 

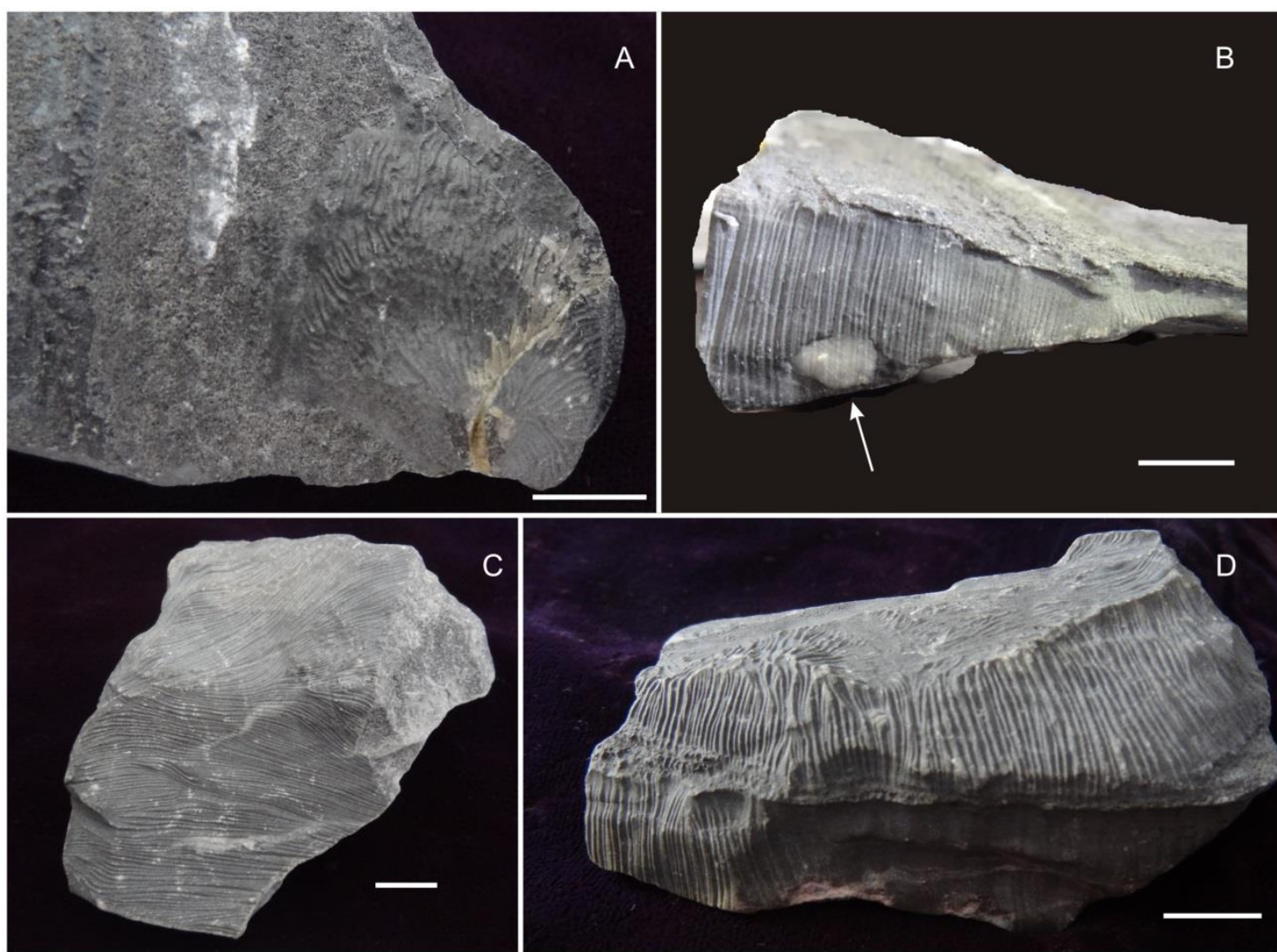

Figura 7. Kinneyia lucianoi. A) Amostra 1683-Pb. B) Amostra 1683-Pb. Notar cristas passando por lente de calcita secundária. C) Amostra 1684-Pb. Notar o padrão de cristas paralelas entre si e os veios diagenéticos de calcita, também cortados pelas cristas. D) Amostra 1685-Pb.

Notar que as mesmas cristas estão presentes na superfície da amostra e na lateral, o que não é esperado para este tipo de estrutura. Escalas: $1 \mathrm{~cm}$.

Além disso, as cristas não se restringem apenas à superfície de acamamento, mas também às faces laterais das amostras (figura 7D).

Seguindo a mesma linha de argumentações desenvolvida acima para $B$. hectoris, é notório que Kinneyia lucianoi não consiste de uma estrutura primária, uma vez que ocorre também em estruturas diagenéticas. Além disso, as cristas,

\section{Bambuites erichsenii}

Sommer (1971) descreveu microfósseis também provenientes de calcários da localidade de Pedro Leopoldo. Estes fósseis foram então descritos como novo gênero e espécie Bambuites erichsenii. São caracterizados pela cor escura, provavelmente de composição carbonosa, mais claros nas bordas, formato vesicular, com tamanho variado entre $70 \mathrm{e}$ $200 \mu \mathrm{m}$ e borda crenulada. O autor atribuiu-Ihes afinidades com algas unicelulares, precipitadoras de calcita, semelhantes ao gênero Valeria, encontrado até então no Mesoproterozoico da Groelândia (Pedersen \& Lam, 1968 apud Sommer, 1971).

A reanálise da lâmina confeccionada por Sommer permitiu observar os espécimes descritos por ele (figuras $8 \mathrm{~A}-\mathrm{D}$ ) e parte das características citadas no trabalho foram observadas, com exceção das bordas mais claras e crenuladas. Ao contrário, este exame diagnósticas desta espécie, ocorrem em diferentes faces das amostras, enquanto que o padrão Kinneyia, profundamente estudado em outras unidades, mostra-se como uma estrutura restrita ao acamamento, não adentrando ao sedimento. Com base no exposto, é possível inferir que Kinneyia lucianoi trata-se de pseudofósseis e não estruturas formadas por ação microbiana sobre sedimentos.

mostrou que o centro das estruturas é ligeiramente mais claro que a borda (figura $8 \mathrm{C}$ ), onde é possível observar a textura granular dos espécimes. Outra inconsistência com a análise original foi que a parede celular contém processos curtos, semelhantes a espinhos, ao invés de parede crenulada. $O$ aspecto crenulado (figura $8 \mathrm{~A}$ ) talvez se deva aos pontos em que tais processos (figuras $8 \mathrm{~A} e$ 8D) se conectam à parede.

A presença de estruturas ao longo da parede, semelhante a processos curtos são condizentes com o gênero Trachyhystrichosphaera Timofeev \& Hermann 1976, emend. Butterfield et al. 1994. No entanto, a análise pormenorizada revelou que estes processos são apenas extensões da parede, não consistindo de processos verdadeiros, podendo ser de origem tafonômica. Outra característica de origem tafonômica é a cor preta, que pode ser explicada pelo fato de os microfósseis terem sido 
submetidos a altas temperaturas em consequência ao metamorfismo ao qual o grupo foi submetido.

De fato, trata-se de microfósseis de parede orgânica, cuja variação de tamanho, a textura e as dobras da parede (figuras $8 \mathrm{~B}-\mathrm{C}$ ) permitem atribuirIhes afinidade com eucariontes. Outros caracteres, como a forma, textura da parede e padrão de dobras, embora pouco nítidas, os aproxima de Leiosphaeridia jacutica Timofeev 1966, emend. Mikhailova \& Jankauskas 1989, bastante comum no Neoproterozoico tardio. Desta forma, a taxonomia de Bambuites erichsenii (Sommer, 1971) passa a ser a seguinte:

\section{Grupo Acritarcha Evitt, 1963}

Leiosphaeridia jacutica (Timofeev, 1966), emend. Mikhailova \& Jankauskas, 1989

Leiosphaeridia warsanofiewii Naumova (Timofeev, 1966)

Kildinella jacutica sp. nov. (Timofeev, 1969, 1982)

K. jacutica Tim. (Timofeev, 1969; Aseeva, 1976)

K. perforata Fanderflitt sp. nov. (Konopleva, Fanderflitt, 1979)

K. cf. jacutiga Tim. (Knoll, 1984)

K. aff. perforata Fanderflitt (Volkova, 1981)

Chuaria circularis Walcott 1899 (Vidal, 1976, 1979; Vidal, Siedlecka, 1983; Vidal, Ford, 1985; Knoll, Calder, 1983; Knoll, 1984)

Leiosphaeridia spp. (Damassa, Knoll, 1986)

Bambuites erichsenii (Sommer, 1971)

Holótipo. IGD Academia Russa de Ciências n.452/1.

Lectótipo. IGD Academia Russa de Ciências n.1821/1.

Descrição. Grande vesícula rígida, com parede mais espessa, densa, fracamente transparente, com dobras lanceoladas pequenas a grandes. Diâmetro de 70 a 800 micrometros. Frequentemente observase o citoplasma colapsado no interior da carapaça. Em comparação com $L$. crassa difere apenas pelo tamanho maior.

Observações. O tamanho de L. jacutica é similar ao dos espécimes mais finos de Chuaria circularis, no sentido mais amplo da diagnose deste último. No entanto, Jankauskas et al. (1989) limitaram o tamanho dos espécimes de Chuaria circularis em 1000 micrômetros e mantiveram as vesículas menores na diagnose de $L$. jacutica. De acordo com Grey (2005), L. jacutica trata-se de uma espécie comum no Ediacarano Inferior da Austrália, típico da biozona pós-glaciação Marinoana.

Material e distribuição. Dezenas de citações no Rifeano e em diferentes regiões vendianas da Rússia (Jankauskas et al., 1989). Formação Browne, Austrália (Cotter, 1977); Quartzito ABC Range, Austrália (Grey, 2005); Formação Murnaroo, Austrália (Arouri et al., 2000); Mudstone Dey Dey, Austrália (Grey, 2005; Willman \& Moczydlowska, 2007); Formação Pertatataka, Austrália (Grey, 2005); Formação Liulaobei, Grupo Huainan, China (Tang et al., 2013); formações Hussar, Kanpa e Steptoe, Grupo Buldya, Austrália (Grey et al., 2011); Grupo Roper, Austrália (Javaux \& Marshall, 2006); Formação Bezymenka, Uplift Turukhan, Rússia (Khomentovsky et al., 2008); Supergrupo Thule, Canadá e Groelândia (Samuelsson et al., 1999); Formação Anyaboni, Grupo Kwahu, Ghana (Couëfé \& Vecoli, 2011); Supergrupo Bylot, Canadá (Hofmann \& Jackson, 1994); Grupo Bambuí (este trabalho).

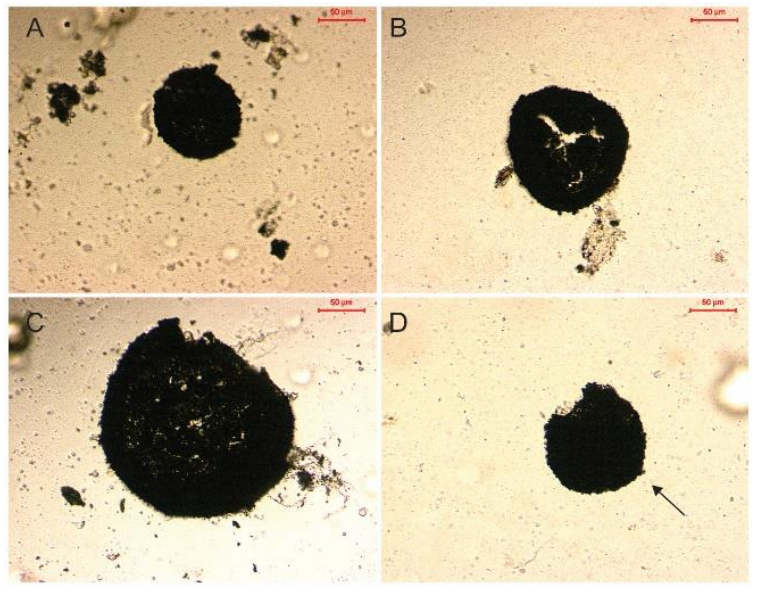

Figura 8. Leiosphaeridia jacutica. A) notar opacidade do exemplar e parede crenulada. B) exemplar parcialmente fragmentado. C) exemplar de grande porte. Notar textura granular no centro do microfóssil. D) Seta indica o que parece ser um curto processo, mas que, após exame detalhado, revelou ser uma feição tafonômica. Escalas: $50 \mu \mathrm{m}$

\section{CONSIDERAÇÕES FINAIS: UM NOVO PANORAMA ACERCA DA PALEOBIOLOGIA DO GRUPO BAMBUÍ}

O conteúdo fóssil do Grupo Bambuí sempre foi considerado esparso e pouco significativo do ponto de vista bioestratigráfico, já que seu registro de vida pretérita consiste principalmente de microbialitos e microfósseis de cianobactérias e bactérias formadoras de esteiras. Estes grupos são sabidamente de pouca aplicação em cronobioestratigrafia e correlações estratigráficas interbacinais (Grotzinger \& Knoll, 1999).

Por outro lado, são exceções os trabalhos de Hidalgo (2007), que revelou a preservação de acritarcos e outras formas incertae sedis típicas do 
início do Ediacarano, e de Warren et al. (2014), que apresentaram a ocorrência do gênero Cloudina, um fóssil-índice para o fim do Neoproterozoico, ao norte de Minas Gerais. Ambos os trabalhos consistem de importantes contribuições para o estabelecimento da idade da base do Grupo Bambuí e para situá-lo no panorama mundial já conhecido para o Neoproterozoico, seja do ponto de vista da evolução da vida, seja do ponto de vista das mudanças paleoambientais ocorridas neste intervalo de tempo (Fairchild et al., 2012).

Completavam o quadro de fósseis do Grupo Bambuí diversas estruturas enigmáticas encontradas ao longo do século passado, cuja afinidade biológica e o valor evolutivo e bioestratigráfico originais propunham uma diversidade de vida maior que o usualmente relatado para o grupo. Essas estruturas, objetos do presente estudo, foram originalmente descritas como palinomorfos e fósseis de algas. No entanto, a partir da reanálise do material depositado no DNPM-RJ sob a luz de conceitos modernos em Paleontologia, conclui-se que, dos quatro táxons identificados, apenas um, Bambuites erichsenii Sommer 1971, permanece válido como fóssil, sendo posto em sinonímia com Leiosphaeridia jacutica Timofeev 1966, emend. Mikhailova \& Jankauskas 1989. Demais táxons foram reavaliados como pseudofósseis.

Quanto ao reconhecimento de Leiosphaeridia jacutica, sua identificação soma mais uma espécie aos acritarcos já identificados, entre eles Trachysphaeridium, Stictosphaera e Protosphaeridium (Cruz \& Lopes, 1992) e Cymatiosphaeroides sp., Cymatiosphaeroides kullingii, Simia sp., Spumosina rubiginosa, Trachyhystrichosphaera aimica, Trachyhystrichosphaera truncata, Trachyhystrichosphaera laminatarium, Vandalosphaeridium sp. e Leiosphaeridia sp. (Hidalgo, 2007). Esta reclassificação dos fósseis do Grupo Bambuí pouco altera o panorama palinológico já conhecido para a unidade, uma vez L. jacutica apresenta ampla distribuição espacial e temporal, portanto, sem valor bioestratigráfico. O único intervalo de tempo em que $L$. jacutica tem valor bioestratigráfico é quando ocorre juntamente com Leiosphaeridia crassa Naumova (1949) emend. Jankauskas et al. 1989 na Biozona ELP (Edicaracan Leiosphere Palynoflora), representativa do intervalo de tempo entre o fim da glaciação Marinoana (ca. $635 \mathrm{Ma}$, Grey, 2005) e o momento da diversificação de acritarcos acantomorfos (ca. $580 \mathrm{Ma}$ ), típicos das palinobiotas de Doushantuo e Pertatataka (Grey, 2005; Huntley et al., 2006). L. crassa não foi identificada no material de Sommer (1971), mas foi identificada por Hidalgo (2007) em amostras da Formação Sete Lagoas. No entanto, a procedência estratigráfica de L. jacutica é incerta. Se a amostra foi coletada em um nível da Formação Sete Lagoas, na bse do Grupo Bambuí, então a ocorrência de $L$. crassa e L. jacutica na mesma unidade poderia apontar, mesmo que de maneira preliminar, para a ocorrência de formas semelhantes à da biozona ELP na Formação Sete Lagoas, sugerindo sua deposição, de fato, após a glaciação Marinoana como proposto por Caxito et al. (2012). Se L. jacutica recuperada por Sommer provier de uma unidade diferente da Formação Sete Lagoas, então trata-se de uma ocorrência sem valor bioestratigráfico, como ocorre em outras unidades do Neoproterozoico. A importância da recuperação de $L$. jacutica em rochas do Grupo Bambuí sinaliza para a necessidade de pesquisas pormenorizadas acerca de microfósseis e sua contextualização no panorama do Neoproterozoico mundial.

\section{AGRADECIMENTOS}

Os autores agradecem ao Departamento Nacional de Produção Mineral no Rio de Janeiro, em especial ao paleontólogo Rodrigo Machado, pela autorização de análise do material; ao Dr. Guilherme R. Romero (IG/UFPA), pela gentil revisão do texto e colaboração para sua melhoria; ao apoio financeiro do Conselho Nacional de Pesquisa e Desenvolvimento Tecnológico - CNPq - através do projeto 401815/2010-2; e à Fundação de Amparo à Pesquisa do Estado de São Paulo - FAPESP - pela concessão de bolsa de doutorado (processo 2001/07203-9), em cujo âmbito o presente estudo foi desenvolvido.

\section{REFERÊNCIAS BIBLIOGRÁFICAS}

Alkmim F.F. \& Martins-Neto M.A. 2012. Proterozoic first-order sedimentary sequences of the São Francisco craton, eastern Brazil. Marine and Petroleum Geology, 33(1): 127-139.

Arouri K., Greenwood P.F., Walter M.R. 2000. Biological affinities of Neoproterozoic acritarchs from Australia: microscopic and chemical characterisation. Organic Geochemistry 31: 75-89.

Billings E. 1872. On some fossils from the primordial rocks of Newfoundland. Canadian Naturalist, 6: 465-479.

Bittencourt J.S., Kuchenbecker M., Vasconcelos A.G., Meyer K.E. 2015. O registro fóssil das coberturas sedimentares do Cráton do São Francisco em Minas Gerais. Revista Geonomos, 23(2): 39-62.

Brasier M., Green O., Shields G. 1997. Ediacarian sponge spicule clusters from southwestern Mongolia and the origins of the Cambrian fauna. Geology, 25(4): 303-306.

Buss L.W. \& Seilacher A. 1994. The Phylum Vendobionta: a sister group of the Eumetazoa?. Paleobiology, 20(01): 1-4.

Butterfield N.J., Knoll A.H., Swett K. 1994. Paleobiology of the Neoproterozoic Svanbergfjellet Formation, Spitsbergen. Fossils \& Strata, 34: $88 \mathrm{pp}$

Caxito F.A., Halverson G.P., Uhlein A., Stevenson R., Dias T.G., Uhlein G.J. 2012. Marinoan glaciation in east central Brazil. Precambrian Research, 200: 38-58. 
Chen J.Y., Bottjer D.J., Davidson E.H., Li G., Gao F., Cameron R.A., Hadfield M.G., Xian D.C., Tafforeau P., Jia Q.J., Sugiyama H., Tang R. 2009. Phase contrast synchrotron X-ray microtomography of Ediacaran (Doushantuo) metazoan microfossils: phylogenetic diversity and evolutionary implications. Precambrian Research, 173(1): 191-200.

Cornish F.G. 1986. The trace-fossil Diplocraterion: Evidence of animal-sediment interactions in Cambrian tidal deposits. Palaios, 1(5): 478-491.

Cotter E. 1977. The Evolution of Fluvial Style, With Special Reference to the Central Appalachian Paleozoic. In: Miall A.D. Fluvial Sedimentology. Dallas Geological Society, Memoir 5 Ancient Fluvial Systems, p. 361-383.

Couëffé R. \& Vecoli M. 2011. New sedimentological and biostratigraphic data in the Kwahu Group (Meso-to NeoProterozoic), southern margin of the Volta Basin, Ghana: Stratigraphic constraints and implications on regional lithostratigraphic correlations. Precambrian Research, 189(1): 155-175.

CPRM - Serviço Geológico do Brasil 2014. Mapa Geológico do Estado de Minas Gerais. Belo Horizonte, Mapa Geológico, escala: 1:1.000.000.

Cruz N.M. \& Lopes J.N. 1992. Microfósseis do Grupo Bambuí, na região de Arcos, Minas Gerais. Anais da Academia Brasileira de Ciências, 64(4): 420.

Fairchild, T.R., Schopf, J.W., Shen-Miller, J., Guimarães, E.M., Edwards, M.D., Langstein, A., Li, X., Pabst, M., Melo-Filho, L.S. 1996. Recent discoveries of Proterozoic microfossils in southcentral Brazil. Precambrian Research, 80: 125-152.

Fairchild T.R., Sanchez E.A.M., Pacheco M.L.A.F., Leme, J.M. 2012 Evolution of Precambrian life in the Brazilian Geological Record. International Journal of Astrobiology, 11(4): 309-323.

Fedonkin M.A. 2003. The origin of the Metazoa in the light of the Proterozoic fossil record. Paleontological Research, 7(1): 941.

Fedonkin M.A., Vickers-Rich P., Swalla B.J., Trusler P., Hall M. 2012. A new metazoan from the Vendian of the White Sea, Russia, with possible affinities to the ascidians. Paleontological Journal, 46(1): 1-11.

Fernandes A.C.S. 1985. Redescrição de Arthraria riachaoensis Maury e comentários sobre a validade como bióglifo. Anais da Academia Brasileira de Ciências, 57(2): 199-201.

Fillion D. \& Pickerili R.K. 1984. On Arthraria antiquata Billings, 1872 and its relationship to Diplocraterion Torell, 1870 and Bifungites Desio, 1940. Journal of Paleontology, 58(3): 683696.

Flügel E. \& Axel M. (Eds.) 2010. Microfacies of carbonate rocks: analysis, interpretation and application. Springer-Verlag, Berlim, 2 a ed, 1006 pp.

Grant S.F.W. 1990. Shell structure and distribution of Cloudina, a potential index fossil for the terminal Proterozoic. American Journal of Science, 290: 261-294.

Grey K. 2005. Ediacaran palynology of Australia. Association of Australasian Palaeontologists, 31: $439 \mathrm{pp}$.

Grey K., Hill A.C., Calver C. 2011. Biostratigraphy and stratigraphic subdivision of Cryogenian successions of Australia in a global context. Geological Society Memoirs, 36(1): 113-134.

Grotzinger J.P. \& Knoll A.H. 1999. Stromatolites in Precambrian carbonates: evolutionary milesposts or environmental dipsticks?. Annual Review of Earth and Planetary Sciences, 27: 313- 358.
Hantzschel W. 1975. Treatise on Invertebrate Paleontology, Part W, Miscellanea, Supplement 1, Trace Fossils and Problematica. University of Kansas Press, USA, 269 pp.

Hidalgo R.L.L. 2007. Vida após as glaciações globais neoproterozóicas: um estudo microfossifífero de capas carbonáticas dos Crátons do São Francisco e Amazônico. Tese de Doutoramento, Instituto de Geociências, Universidade de São Paulo, 195 pp.

Hofmann H.J. \& Jackson G.D. 1994. Shale-facies microfossils from the Proterozoic Bylot Supergroup, Baffin Island, Canada. Memoir of The Paleontological Society, 37(suplem. 68, n.4): 1-39.

Huntley J.W., Xiao S., Kowalewski M. 2006. 1.3 billion years of acritarch history: an empirical morphospace approach. Precambrian Research, 144(1): 52-68.

Jankauskas T.V., Mikhailova N. S., Hermann T.N. 1989. Mikrofossilii dokembriya SSSR [Precambrian Microfossils of the USSR]. Trudy Instituta Geologii i Geochronologii Dokembria SSSR Akademii Nauk, Leningrad, p. 188. Traduzido para o Português.

Javaux E.J. \& Marshal C.P. 2006. A new approach in deciphering early protist paleobiology and evolution: Combined microscopy and microchemistry of single Proterozoic acritarchs. Review of Palaeobotany and Palynology, 139(1): 115.

Khomentovsky, V.V., Nagovitsin, K.E., Postnikov, A.A. 2008. Mayanian (1100-850 Ma) - Prebaikalian Upper Riphean of Siberia. Russian Geology and Geophysics, 49: 1-22.

Knaust D. 2013. The ichnogenus Rhizocorallium: classification, trace makers, palaeoenvironments and evolution. EarthScience Reviews, 126: 1-47.

Knoll A.H. 2003. The geological consequences of evolution. Geobiology, 1: 3-14.

Kuchenbecker M. 2014. Relações entre coberturas do Cráton do São Francisco e bacias situadas em orógenos marginais: o registro de datações $\mathrm{U}-\mathrm{Pb}$ de grãos detríticos de zircão e suas implicações geotectônicas. Tese de Doutorado, Instituto de Geociências, Universidade Federal de Minas Gerais, 175 pp.

Leonov M.V. 2007. Comparative taphonomy of Vendian genera Beltanelloides and Nemiana: taxonomy and lifestyle. In: Vickers-Rich P. \& Komarower P (Eds.) The Rise and Fall of the Ediacaran Biota. Special Publications of Geological Society, 286, London, p. 259-267.

Li C.W., Chen J.Y., Hua T.E. 1998. Precambrian sponges with cellular structures. Science, 279: 879-882.

Maury C.J. 1929. Calcareo fossilifero de Bom Jesus da Lapa, Bahia. Rio de Janeiro, Serviço Geológico e Mineralógico do Brasil, Monografia 7, p. 46-53.

Mikhailova N.S. \& Jankauskas T.V. 1989. Descrição Sistemática de Microfósseis, Leiosphaeridia jacutiga. In: Jankauskas T.V. Microfósseis Pré-Cambrianos da URSS. Editora Ciência, 3, Moscou, p. 77-78. Traduzido para o Português.

Miller A. 1875. Some new species of fossils from the Cincinnati Group and remarks upon some described forms. Journal of Science, 2: 349-355.

Mills D.B. \& Canfield D.E. 2014. Oxygen and animal evolution: did a rise of atmospheric oxygen "trigger" the origin of animals?. BioEssays, 36(12): 1145-1155.

Monty C.L. 1981. Spongiostromate vs. porostromate stromatolites and oncolites. In: Bertrand-Sarfati J. (Ed.) Phanerozoic stromatolites. Springer, Berlin Heidelberg, pp. 1-4. 
Morris S.C. \& Caron J.B. 2012. Pikaia gracilens Walcott, a stemgroup chordate from the Middle Cambrian of British Columbia. Biological Reviews, 87(2): 480-512.

Pedersen, K. \& Lam, J. 1968. Precambrian organic compounds from the Ketilidian of South West Greenland, part II. Meddeleser om Grønland, 185(6): 1-16.

Pia J. 1927. Thallophyta. In: Hirmer M. (Ed.) Handbuch der Paläobotanik. München, 1, p. 31-136.

Porada H. \& Bouougri E. 2007. "Wrinkle structures" - a critical review. In: Schieber J., Bose P., Eriksson, P.G., Banerjee S., Sarkar S., Altermann W., Catuneanu O. (Eds.) Atlas of microbial mat features preserved within the siliciclastic rock record. Amsterdã, Elsevier, p. 135-144.

Reis H.L. \& Suss J.F. 2016. Mixed carbonate-siliciclastic sedimentation in forebulge grabens: an example from the Ediacaran Bambuí Group, São Francisco Basin, Brazil. Sedimentary Geology, 339: 83-103.

Rodrigues J.B. 2008. Proveniência de sedimentos dos grupos Canastra, Ibiá, Vazante e Bambuí - Um estudo de zircões detríticos e Idades Modelo SM-Nd. Tese de Doutorado, Instituto de Geociências, Universidade de Brasília, 128 pp.

Samuelsson J., Dawes P.R., Vidal G. 1999. Organic-walled microfossils from the Proterozoic Thule Supergroup, Northwest Greenland. Precambrian Research, 96(1): 1-23.

Sanchez E.A.M. 2014. Microbialitos e microfósseis da Formação Sete Lagoas, Neoproterozoico, Brasil: implicações geomicrobiológicas em um contexto de mudanças climáticas e evolutivas. Tese de Doutorado, Instituto de Geociências, Universidade de São Paulo, 299 pp.

Schopf J.W. 1992. The oldest fossils and what they mean. In: Schopf J.W. (Ed.) The Major Events in the History of Life. Jones and Bartlett Publishers, Boston, p. 33.

Seilacher A. 1992. Vendobionta and Psammocorallia: lost constructions of Precambrian evolution. Journal of the Geological Society, 149(4): 607-613.

Seilacher A. 2001. Concretion morphologies reflecting diagenetic and epigenetic pathways. Sedimentary Geology, 143(1): 4157.

Seilacher A. 2007. The nature of vendobionts. Special Publications of Geological Society of London, 286(1): 387-397.

Seilacher A., Grazhdankin D., Legouta A. 2003. Ediacaran biota: The dawn of animal life in the shadow of giant protists. Paleontological Research, 7(1): 43-54.

Sommer F.W. 1970. Bióglifos do Calcário Bambuí, de Pedro Leopoldo, Estado de Minas Gerais. Anais da Academia Brasileira de Ciências, 42(3): 453-458.

Sommer F.W. 1971. Microfósseis do Calcário Bambuí, de Pedro Leopoldo, Estado de Minas Gerais. Anais da Academia Brasileira de Ciências, 43(1): 135-139.

Sommer F.W. 1981. Oncólitos do Calcário Bambuí de Minas Gerais. Anais da Academia Brasileira de Ciências, 53(4): 785791.

Sommer F.W. 1982. Mais oncólitos do Calcário Bambuí de Minas Gerais. Anais da Academia Brasileira de Ciências, 54(2): 343349.
Sperling E.A., Frieder C.A., Raman A.V., Girguis P.R., Levin L.A., Knoll A.H. 2013. Oxygen, ecology, and the Cambrian radiation of animals. Proceedings of the National Academy of Sciences, 110(33): 13446-13451.

Steiner M., Mehl D., Reitner J., Erdtmann B.D. 1993. Oldest entirely preserved sponges and other fossils from the lowermost Cambrian and a new facies reconstruction of the Yangtze Platform (China). Berliner Geowiss. Abh., 3: 293-329.

Tafforeau P., Boistel R., Boller E., Bravin A., Brunet M., Chaimanee Y., Cloetens P., Feist M., Hoszowska J., Jaeger J, Kay R.F., Lazzari V., Marivaux L., Nel A., Nemoz C., Thibault X., Vignaud P., Zabler S. 2006. Applications of X-ray synchrotron microtomography for non-destructive $3 \mathrm{D}$ studies of paleontological specimens. Applied Physics A, 83(2): 195-202.

Tang Q., Pang K., Xiao S., Yuan X., Ou Z., Wan B. 2013. Organicwalled microfossils from the early Neoproterozoic Liulaobei Formation in the Huainan region of North China and their biostratigraphic significance. Precambrian Research, 236: 157-181.

Tang T., Zhang J., Jiang X. 1978. Discovery and significance of the Late Sinian fauna from Western Hunan and Hubei. Acta Stratigraphica Sinica, 2: 32-45.

Timofeev B.V. 1966. Microphytological investigations of ancient formations. Laboratory of Precambrian Geology and Geochronolology of Academia of Science of USSR, Leningrad Nauka, p. 1-47.

Timofeev B.V. \& Hermann T.N. 1976. Dokembrijskaya mikrobiota Lakhandinskoj svity. In: Sokolov B.S. (Ed.) Paleontologiya Dokembriya i Rannego Kembriya. Nauka, Leningrad, p. 37147. Traduzido para o Português.

Tiwari, M., Pant, C.C., Tewari, V.C. 2000. Neoproterozoic sponge spicules and organic walled microfossils from the Gangolihat Dolomite, Lesser Himalaya, India. Current Science, 79 (5), 651-654.

Warren L.V., Quaglio F., Riccomini C., Simões M.G., Poiré D.G., Strikis N.M., Anelli L.E., Strikis P.C. 2014. The puzzle assembled: Ediacaran guide fossil Cloudina reveals an old proto-Gondwana seaway. Geology,42(5): 391-394.

Willman S. \& Moczydłowska M. 2007. Wall ultrastructure of an Ediacaran acritarch from the Officer Basin, Australia. Lethaia, 40(2): 111-123.

Xiao S. \& Laflamme M. 2009. On the eve of animal radiation: phylogeny, ecology and evolution of the Ediacara biota. Trends in Ecology and Evolution, 24(1): 31-40.

Zhang Y. 1989. Multicellular thallophytes with differentiated fossils from Late Proterozoic phosphate deposits in South China. Lethaia, 22: 113-132.

Zhang Y.; Yin L.; Xiao S.; Knoll A.H. 1998. Permineralized fossils from the terminal Proterozoic Doushantuo Formation, south China.Memoir -The Paleontological Society, 50(4): 1-52.

Zucatti Da Rosa A.L. 2005. Evidências de vida no Ediacarano Inferior da Bacia do Itajaí, SC. Dissertação de Mestrado, Centro de Ciências Exatas e Tecnológicas, Universidade do Vale do Rio dos Sinos, 56 pp. 